\title{
AKR1B10 expression in benign prostatic hyperplasia and its related mechanism
}

\author{
WENJING XU ${ }^{1}$, YA GAO ${ }^{2}$, JIAQI ZHANG ${ }^{1}$, RONG ZHANG $^{1}$ and QIHUA CHEN $^{3}$ \\ ${ }^{1}$ First Clinical College of Chinese Medicine, Hunan University of Chinese Medicine, \\ Changsha, Hunan 410208; Departments of ${ }^{2}$ Proctology and ${ }^{3}$ Surgery and Male Disease, \\ The First Hospital of Hunan University of Chinese Medicine, Changsha, Hunan 410007, P.R. China
}

Received October 17, 2019; Accepted November 24, 2020

DOI: $10.3892 / \mathrm{ol} .2021 .12944$

\begin{abstract}
The aim of the present study was to investigate the expression of aldo-keto reductase family 1 member B10 (AKR1B10) in benign prostatic hyperplasia (BPH) and its related mechanism. In total, $142 \mathrm{BPH}$ patients admitted from March 2017 to March 2019 at the First Hospital of Hunan University of Chinese Medicine and 140 healthy people undergoing physical examination were selected as the research subjects. The clinical value of AKR1B10 in BPH was analyzed. Twenty clean SD rats were selected, and 10 were selected to establish the prostate hyperplasia model, while the remaining 10 were set as the control group. Ten days after the model was established, AKR1B10 and NF- $\kappa \mathrm{B}$ expression in prostate tissues of rats in both groups was detected by PCR and immunohistochemistry. The primary cells in prostate hyperplasia were cultured, and then they were transfected with AKR1B10 to observe the changes of cell biological behavior. AKR1B10 and $N F-\kappa B$ mRNA significantly increased in peripheral blood of $\mathrm{BPH}$ patients and prostate tissue of $\mathrm{BPH}$ model rats $(\mathrm{P}<0.001)$, and AKR1B10 had good diagnostic value for $\mathrm{BPH}$ $(\mathrm{P}<0.001)$. In addition, it was positively correlated with PSA, EGF, IL-6 and TNF- $\alpha(\mathrm{P}<0.001)$. After transfection with AKR1B10-inhibitor, it was revealed that the proliferation of prostate hyperplasia cells decreased, while the apoptosis of prostate hyperplasia cells increased and the $\mathrm{NF}-\kappa \mathrm{B}$ protein expression decreased $(\mathrm{P}<0.001)$. Collectively, high expression of AKR1B10 in $\mathrm{BPH}$ promoted the proliferation of prostate cells and reduced their apoptosis, and the mechanism may be through regulation of $\mathrm{NF}-\kappa \mathrm{B}$.
\end{abstract}

Correspondence to: Dr Qihua Chen, Department of Surgery and Male Disease, The First Hospital of Hunan University of Chinese Medicine, 95 Shaoshan Road, Changsha, Hunan 410007, P.R. China

E-mail: hncscqh@163.com

Key words: aldo-keto reductase family 1 member $\mathrm{B} 10, \mathrm{NF}-\kappa \mathrm{B}$, benign prostatic hyperplasia, proliferation, apoptosis

\section{Introduction}

Benign prostatic hyperplasia (BPH) is prostate hyperplasia, which is one of the most common diseases of middle-aged and elderly males clinically at present (1). Currently, the global lifetime prevalence rate of $\mathrm{BPH}$ is $26.2 \%$, and the morbidity in urban areas is markedly higher than that in rural areas (2). However, with the change of disease incidence, a great number of studies have revealed that BPH is diagnosed at younger ages $(3,4)$. BPH is a benign disease with a slow progression; however, it is possible to have a worsening crisis if it is not properly controlled (5). It can cause bladder neck contracture, and repeated attacks cause urinary tract infection and lower urinary tract obstruction. Symptoms of BPH patients include obvious urination abnormalities and urinary incontinence (6). More serious cases may lead to prostate cancer, endangering the life and health of patients (7). At present, conservative treatment is mainly adopted for BPH clinically, and the most commonly used drugs include $5 \alpha$-reductase inhibitor, $\alpha 1$-receptor blocker and $\mathrm{M}$ receptor antagonist, which can achieve certain efficacy for moderate and severe cases (8). However, patients who fail to receive conservative treatment and meet surgical criteria select surgery for treatment. After prostatectomy, patients are likely to experience dysuria, paruria, epididymitis, and some may experience erectile dysfunction, interfering with their sexual activity, which seriously affects their quality of life (9). Therefore, timely treatment in the early stage of BHP progression has a crucial impact on the prognosis of patients. However, the diagnosis of $\mathrm{BHP}$ requires a series of examinations including rectal digital examination, B-ultrasound, residual urine and urinalysis (10) that are not conducive to early diagnosis. Therefore, fully understanding the pathogenesis of BPH is the key to prevent and treat BPH in the future.

Aldo-keto reductase family 1 member B10 (AKR1B10) is located in the human chromosome $7 \mathrm{q} 33$ region. It can encode a protein consisting of 316 amino acid residues, belonging to a member of the aldo-keto reductase superfamily (11). To date, research on AKR1B10 has mainly focused on liver and breast cancer. Research has revealed that the mRNA level of AKR1B10 had a certain predictive value for the recurrence of hepatitis B virus-related liver cancer patients, and the positive rate of AKR1B10 in red blood cells of breast cancer patients 
was markedly higher than that of healthy individuals $(12,13)$. However, its influence on BPH remains unclear. AKR1B10 is a secretory protein belonging to a lysosome-mediated non-classical protein-secretion pathway (14). Khan et al (15) considered that the aberrant expression of nonclassical secretory proteins may be the key to $\mathrm{BPH}$. Therefore, it was theorized that AKR1B10 may be a key gene affecting BPH, with marked significance for future clinical diagnosis and treatment. In order to verify this theory, the present study provided a reliable basis for future clinical study by exploring the effect and mechanism of AKR1B10 in BPH.

\section{Materials and methods}

General data. In total, 142 BPH patients $(51.2 \pm 11.6$ years old) and 140 healthy individuals (50.8 \pm 8.9 years old) undergoing physical examination from March 2017 to March 2019 were selected as the research subjects. BPH patients were selected as the research group and healthy people undergoing physical examination were regarded as the control group. This experiment was approved by the Ethics Committee of the First Hospital of Hunan University of Chinese Medicine. All the aforementioned subjects signed informed consent forms.

Inclusion and exclusion criteria. The inclusion criteria were as follows: i) patients aged 20-70; ii) patients conforming to the clinical manifestation of BPH and diagnosed with BPH after examination at the First Hospital of Hunan University of Chinese Medicine; iii) patients with complete medical case data; and iv) patients who agreed to cooperate and participate in the investigation and study of the First Hospital of Hunan University of Chinese Medicine. The exclusion criteria were as follows: i) patients with tumor, cardiovascular and cerebrovascular diseases, as well as other autoimmune and infectious diseases; ii) patients with liver and renal insufficiency due to organ failure; iii) patients with gastrointestinal dysfunction; iv) patients without neurogenic bladder dysfunction; v) diabetics; vi) patients who received surgery, radiotherapy or chemotherapy, or drugs that affect the function of bladder exfoliation or cause LUTS within half a year before admission; vii) patients with nervous system diseases; viii) patients with enuresis; ix) patients with a drug allergy; $x$ ) patients with physical disabilities unable to take care of themselves, bedridden; xi) patients with mental disorders and low treatment compliance; xii) patients transferred from one hospital to another. Inclusion and exclusion criteria in the control group were as follows: i) all the results from the physical examination at the First Hospital of Hunan University of Chinese Medicine were normal; ii) no previous major medical history; iii) patients who agreed to cooperate and participate in the investigation and study of the First Hospital of Hunan University of Chinese Medicine.

Animal data. Twenty clean-grade 6-week-old male Srague-Dawley rats were used as experimental subjects and purchased from Beijing Charles River Laboratory Animal Technology Co., Ltd. with certification number SCXK (Beijing) 2016-0011. weighing $(210 \pm 20) \mathrm{g}$, were kept in cages ( 5 in one cage) and maintained at a temperature of $29 \pm 2{ }^{\circ} \mathrm{C}$, a humidity of $40-50 \%$ and a 12 -h light/dark cycle. Food and water were provided ad libitum. This experiment was approved by the Animal Ethics Committee of the First Hospital of Hunan University of Chinese Medicine.

Cell data. Human prostate hyperplasia cells line BPH-1 was purchased from BeNa Culture Collection (BNCC339850). It was adjusted to $1 \times 10^{5}$ cells $/ \mathrm{ml}$, transfected and cultured at $37^{\circ} \mathrm{C}$ under $95 \%$ oxygen and $5 \% \mathrm{CO}_{2}$.

\section{Methods}

Reverse transcription-quantitative $(R T-q) P C R$ detection method. In total, $5 \mathrm{ml}$ fasting venous blood was drawn from patients in the research group and the control group before treatment. The blood was left $30 \mathrm{~min}$ at room temperature and centrifuged $10 \mathrm{~min}\left(1,505 \mathrm{x} \mathrm{g}, 4^{\circ} \mathrm{C}\right)$, and the upper serum was obtained and stored in a refrigerator at $-80^{\circ} \mathrm{C}$ for later use. The PCR method was used to detect the expression of AKR1B10 and $N F-\kappa B$ in the serum of patients. The collected serum was extracted with an EasyPure miRNA kit (cat. no. ER601-01; Beijing TransGen Biotech Co., Ltd.) according to the manufacturer's instructions, and purity, concentration and integrity of the extracted total RNA was tested by ultraviolet spectrophotometer and agarose gel electrophoresis. The total RNA was reverse transcribed using TransScript ${ }^{\circledR}$ miRNA RT Enzyme Mix and 2xTS miRNA Reaction Mix (cat. no. AQ321-01; Beijing TransGen Biotech Co., Ltd.), and the operation steps were strictly in accordance with the manufacturer's kit. Then, PCR amplification was carried out. The PCR reaction system was as follows: $1 \mu \mathrm{l}$ cDNA, $0.4 \mu \mathrm{l}$ each of upstream and downstream primers, $10 \mu 1$ 2xTransTaq ${ }^{\circledR}$ Tip Green qPCR SuperMix, $0.4 \mu \mathrm{l}$ Passive Reference Dye (50X), $\mathrm{ddH}_{2} \mathrm{O}$ supplemented to $20 \mu \mathrm{l}$. The PCR reaction conditions were as follows: Pre-denaturation at $94^{\circ} \mathrm{C}$ for $30 \mathrm{sec}$, denaturation at $94^{\circ} \mathrm{C}$ for $5 \mathrm{sec}$, annealing at $60^{\circ} \mathrm{C}$ for $30 \mathrm{sec}, 40$ cycles in total. Each sample was assessed in triplicate, and the experiment was carried out 3 times. GAPDH was used as an internal reference and the $2^{-\Delta \Delta c q}$ method was used for data analysis (16). Primer sequences are presented in Table I.

Detection of serum markers. Prostate specific antigen (PSA), epidermal growth factor (EGF), interleukin (IL)-6 and tumor necrosis factor (TNF)- $\alpha$ in peripheral blood of patients in the research group were detected. PSA was tested by the laboratory of the First Hospital of Hunan University of Traditional Chinese medicine and EGF, IL- 6 and TNF- $\alpha$ were measured through enzyme-linked immunosorbent assay (ELISA). The EGF kit (cat. no. EH0009) was provided by Wuhan Fine Biotech Co., Ltd., the IL-6 kit (cat. no. SEKH-0013) was purchased from Solarbio Life Sciences, and the TNF- $\alpha$ kit (cat. no. JLC7047) was purchased from Shanghai Jingkang Bioengineering Co., Ltd., and were used according to the manufacturer's instructions.

Diagnostic value prediction. The levels of AKR1B10 and $\mathrm{NF}-\mathrm{kB}$ of both groups were analyzed by ROC curve, and the area under curve (AUC) and the sensitivity and specificity of the two methods for predicting BPH were analyzed.

Modeling method. Twenty rats were randomized into two groups $(n=10)$, and one group was employed as the normal group and the other group was used as the model group to carry 
Table I. Primer sequences.

Reverse

Forward

\begin{tabular}{lll}
\hline AKR1B10 & CAACACGTTACAGGCCCTCC & ACCAGCACGCATTGTTGAGA \\
NF- $\mathrm{BB}$ & TGAGAAGAGGGAGAGCAAGGAAGTC & ACAGAAGCAGGCTGGAGGTAAGG \\
GAPDH & GCGTCAAAGGTGGAGGAGTG & TCAAGAAGGTGGTGAAGCAGG
\end{tabular}

AKR1B10, aldo-keto reductase family 1 member B10.

out BPH modeling; the method was carried out according to a study from Ishola et al (17): $10 \%$ chloral hydrate was injected intraperitoneally at $350 \mathrm{mg} / \mathrm{kg}$ for anesthesia. After complete anesthesia, the rat hair was removed; after routine disinfection, the abdominal cavity was opened, bilateral testicles were removed through the scrotum, and the skin was sutured after ligation and hemostasis at the stump. Rats recovered on their own 7 days after castration, and testosterone propionate ( $5 \mathrm{mg} / \mathrm{kg} /$ time) was injected subcutaneously on day 8 , once a day, for 28 days. On day 29, the rats were anesthetized by intraperitoneal injection of chloral hydrate (as aforementioned) and then euthanized by cervical dislocation.

Immunohistochemical detection method. The prostate tissues of the rats were obtained after they were sacrificed, and one part was fixed in $4 \%$ paraformaldehyde $\left(4^{\circ} \mathrm{C}, 24 \mathrm{~h}\right)$ and the other part was frozen in liquid nitrogen. Then, routine sampling, dehydration, paraffin embedding with $4-\mu \mathrm{m}$ thick sections, and IHC staining $\left(37^{\circ} \mathrm{C}, 1-2 \mathrm{~h}\right)$ were carried out (cytoplasmic staining; AKR1B10 monoclonal antibody; 1:500; cat. no. H00057016; Abnova). The pathological changes of tissues were observed under an optical microscope and images were captured for analysis. Positive cell markers were revealed in tissue sections with pale yellow to tan cells. The staining intensity was scored based on the staining characteristics of most cells (the staining depth was compared with the background staining): 0 for non-staining, 1 point for pale yellow, 2 points for yellow-brown and 3 for tan. The percentage of positive cells referred to the average number of positive cells in 3 fields (x200) of certain cells: $0-5 \%$ was $0,6-25 \%$ was 1 point, $26-50 \%$ was 2 points, $51-75 \%$ was 3 points, and $>75 \%$ was 4 points.

Western blot detection methods. The total protein was extracted from frozen prostate tissue by lysis method (cat. no. R0010; Solarbio Life Sciences), and its concentration was assessed by BCA method and adjusted to $4 \mu \mathrm{g} / \mu \mathrm{l}$. The protein was separated by $12 \%$ SDS-PAGE electrophoresis and then transferred to a PVDF membrane (Molecular weight standard: Lanes 1, 3, 5 and 7; calf liver lysate: Lanes 2, 4, 6 and 8; loading amount per lane $20 \mu \mathrm{g}$; at $4^{\circ} \mathrm{C}$ for $10 \mathrm{~min}$ ). The membrane was stained with $0.2 \%$ Ponceau $\mathrm{S}$ working solution for $10 \mathrm{~min}$ at $4^{\circ} \mathrm{C}$ ), immersed $5 \mathrm{~min}$ in PBST and then washed, blocked for $2 \mathrm{~h}$ at $25^{\circ} \mathrm{C}$ with $5 \%$ skimmed milk powder, and finally sealed overnight at $4^{\circ} \mathrm{C}$ after adding caspase-3 (1:1,000; cat. no. R-1344-100; Biosensis, Ltd.), caspase-9 (1:1,000; cat. no. 3016-30T; BioVision, Inc.), and GPD1 (1:1,000; cat. no. H00002819-A01; Abnova) primary antibodies.
Subsequently, after it was washed to remove the primary antibody, horseradish peroxidase-labeled goat anti-rabbit secondary antibody (1:5,000) was added (cat. no. A-11034; Thermo Fisher Scientific, Inc.), and the protein was incubated for $1 \mathrm{~h}$ at $37^{\circ} \mathrm{C}$. Then, the membrane was rinsed 3 times with PBS, 5 min each time. The protein bands on the membrane were developed in a dark room using an enhanced chemiluminescence reagent (product no. BL523B; Biosharp Life Sciences), and the excess liquid on the membrane was absorbed with a filter paper. The luminescent protein bands were scanned and the gray value was analyzed using Quantity One (v4.6.6; Bio-Rad Laboratories, Inc.). The relative expression of each protein was calculated as follows: Relative protein expression=the gray value of the target protein band/the $\beta$-actin protein band.

Cell culture. The BPH-1 cell line was placed in a culture medium containing 90\% RPMI-1640 medium+10\% FBS and cultured at $37^{\circ} \mathrm{C}$ and $5 \% \mathrm{CO}_{2}$. When the adherent growth and fusion reached $85 \%, 25 \%$ pancreatin was added for digestion. Then, the BPH-1 cell line was placed in a culture medium for continuous culture to complete passage. The concentration of the primer sequences was $10 \mu \mathrm{mol} / 1$, and AKR1B10-mimics (overexpression sequence: Forward, 5'-CGGGGTACCAGATTC AACCAAAGCCAACTCATC-3' and reverse, 5'-CCGCTC GAGGTAGAAGTCTCACGTCCTGCTCTC-3'); AKR1B10mimics-NC (forward, 5'-CCAACTTTTGGCTGTGTTGAA TTTGAAGAGTGAGCATGAACAAGCAGAAACTCCAAT GATAC-3' and reverse, 5'-GTATCATTGGAGTTTCTGCTT GTTCATGCTCACTCTTCAAATTCAACACAGCCAAAA GTTGG-3'); and AKR1B10-inhibitor (inhibitory expression sequence: Forward, 5'-CGGGGTACCATGATGGAC TTGGAGCTGC-3' and reverse, 5'-CCGCTCGAGCTAGTT TTTCTTAACATCTGGCTTC-3'); AKR1B10-inhibitor-NC (forward, 5'-CAACAGAGAGCAGGACGTGAGACT-3' and reverse, 5'-GCATCTTGGCTT TGGTACTGAGCTC-3') were used to transfect cells with a Lipofectamine 2000 kit (cat. no. 11668019; Thermo Fisher Scientific, Inc.), and the operation steps were strictly carried out in accordance with the kit instructions. The primer sequences were designed by Thermo Fisher Scientific, Inc. and their size was $\sim 1,100 \mathrm{bp}$. The transfection temperature was $37^{\circ} \mathrm{C}$, and $36 \mathrm{~h}$ after transfection, the endogenous peroxidase was cleared by hydrogen peroxide disinfector (PCR laboratory hydrogen peroxide disinfector; Shenzhen Runlian Huanbao Technology Co., Ltd.).

Cell Counting Kit-8 (CCK-8) detection. Cells were collected $24 \mathrm{~h}$ after transfection, adjusted to $4 \times 10^{6}$ cells/well and 
Table II. Comparison of general data between research group and control group [n (\%)].

\begin{tabular}{|c|c|c|c|c|}
\hline & Research group $(n=142)$ & Control group $(n=140)$ & $\operatorname{tor} \chi^{2}$ & P-value \\
\hline \multirow[t]{2}{*}{ Age (years) } & & & 0.744 & 0.458 \\
\hline & $56.6 \pm 7.6$ & $57.3 \pm 8.2$ & & \\
\hline \multirow[t]{2}{*}{ BMI $\left(\mathrm{kg} / \mathrm{cm}^{2}\right)$} & & & 0.918 & 0.360 \\
\hline & $24.62 \pm 2.16$ & $24.87 \pm 2.41$ & & \\
\hline Smoking & & & 0.919 & 0.338 \\
\hline Yes & $95(66.90)$ & $86(61.43)$ & & \\
\hline No & $47(33.10)$ & $54(38.57)$ & & \\
\hline Drinking & & & 0.689 & 0.407 \\
\hline Yes & $92(64.79)$ & $84(60.00)$ & & \\
\hline No & $50(35.21)$ & $56(40.00)$ & & \\
\hline Exercise habits & & & 0.982 & 0.322 \\
\hline Yes & $22(15.49)$ & $28(20.00)$ & & \\
\hline No & $120(84.51)$ & $112(80.00)$ & & \\
\hline Place of residence & & & 1.735 & 0.188 \\
\hline Cities and towns & $118(83.10)$ & $124(88.57)$ & & \\
\hline Countryside & $24(16.90)$ & $16(11.43)$ & & \\
\hline Nationality & & & 0.13 & 0.719 \\
\hline Han & $138(97.18)$ & $135(96.43)$ & & \\
\hline Minority & $4 \quad(2.82)$ & $5(3.57)$ & & \\
\hline Family medical history & & & 1.151 & 0.283 \\
\hline Yes & $36(25.35)$ & $28(20.00)$ & & \\
\hline No & $106(74.65)$ & $112(80.00)$ & & \\
\hline
\end{tabular}

inoculated on 96-well plates. Then, after being cultured for 0 , 24, 48 and 72 h, $10 \mu$ l CCK-8 (product no. BS350B; Biosharp Life Sciences) solution and $90 \mu \mathrm{l}$ basic medium (DMEM) were added to each well, and cultured for $2 \mathrm{~h}$ at $37^{\circ} \mathrm{C}$. Finally, the OD values in each group were measured at an absorbance of $450 \mathrm{~nm}$ using an enzyme reader.

Flow cytometry. The transfected cells were digested with $0.25 \%$ trypsin, washed twice with PBS, added using $100 \mu 1$ binding buffer and prepared into $1 \times 10^{6}$ cells $/ \mathrm{ml}$ suspension. Annexin V-FITC and PI (product no. 40302ES20; Shanghai Yeasen Biotechnology Co., Ltd.) used according to the manufacturer's instructions, were sequentially added, and incubated $5 \mathrm{~min}$ at room temperature under dark conditions. Detection was performed using a FC500MCL flow cytometer and CytExpert software (version 2.0; Beckman Coulter, Inc.). FlowJo version 10.0 (Tree Star, Inc.) was also used for analysis. The experiment was repeated 3 times and data were averaged.

Outcome measures. The outcome measures were as follows: i) the AKR1B10 and NF- $\kappa$ B levels in peripheral blood and the levels of PSA, EGF, IL- 6 and TNF- $\alpha$ in the two groups; ii) the correlation between AKR1B10 and PSA, EGF, IL-6, TNF- $\alpha$ in the research group; iii) the AKR1B10 and NF- $\mathrm{BB}$ levels in peripheral blood and prostate tissue of rats; iv) the proliferation and apoptosis of transfected cells and the NF- $\kappa$ B protein expression.
Statistical analysis. The results were analyzed by SPSS 24.0 (Shanghai Yuchuang Network Technology Co., Ltd.) and all graphical results were drawn using GraphPad 8 (Shenzhen Qiruitian Software Technology Co., Ltd.). The counting data were expressed in the form of a rate, and the chi-square test was used for comparison between groups. The measurement data were expressed in the form of the mean \pm standard deviation (SD). Inter-group comparisons were analyzed by Mann-Whitney U test, multi-group comparisons were assessed by one-way ANOVA and LSD post hoc test, and multiple time-points were compared using repeated measures ANOVA and Bonferroni post hoc test. The diagnostic predictive value was analyzed by receiver operating characteristic (ROC) curve, and Pearson correlation coefficient was used for correlation analysis. $\mathrm{P}<0.050$ was considered to indicate a statistically marked difference.

\section{Results}

Comparison of general data. There was no obvious difference in age, BMI, smoking, drinking, exercise habits, place of residence, nationality and family medical history between the research group and the control group ( $\mathrm{P}>0.050$; Table II)

Comparison of $A K R 1 B 10$ and $N F-\kappa B$ expression in peripheral blood. AKR1B10 and NF- $\mathrm{KB}$ in the peripheral blood of the research group were markedly higher than those of the control group $(\mathrm{P}<0.001)$. ROC curve analysis revealed that when 

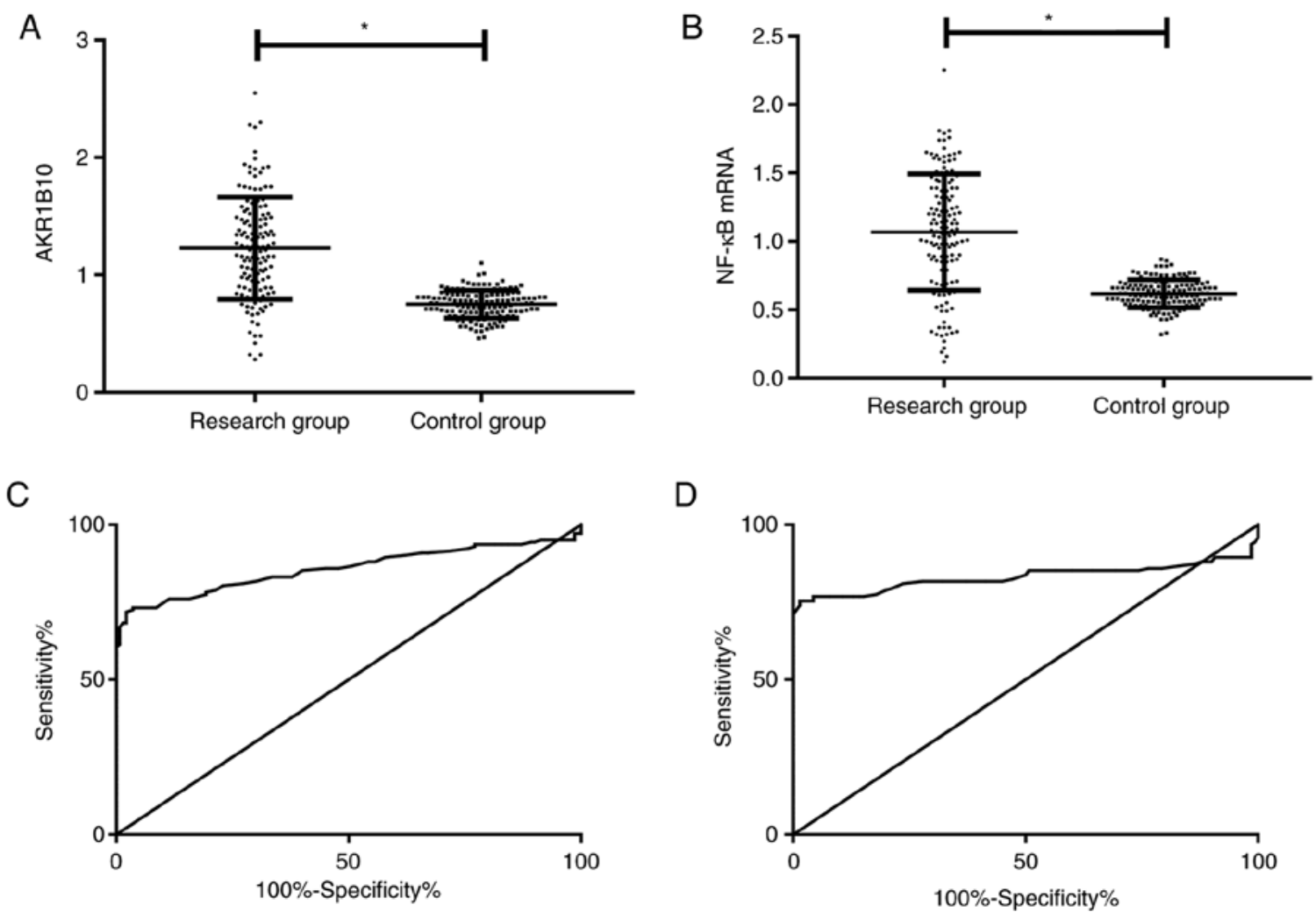

Figure 1. Effects of AKR1B10 and NF-кB in peripheral blood. (A) The AKR1B10 expression in the peripheral blood was compared. It was revealed that its level in the research group was markedly higher than that in the control group ( $\left.{ }^{*} \mathrm{P}<0.001\right)$. (B) The NF- $\mathrm{BB}$ expression in the peripheral blood is compared. It was revealed that its level in the research group was markedly higher than that in the control group ( $\mathrm{P}<0.001)$. (C) ROC curve analysis of AKR1B10 for predicting BPH occurrence. (D) ROC curve analysis of NF- $\mathrm{BB}$ mRNA for predicting BPH occurrence. AKR1B10, aldo-keto reductase family 1 member B10; $\mathrm{BPH}$, benign prostatic hyperplasia; $\mathrm{ROC}$, receiver operating characteristic.

Table III. Diagnostic values of AKR1B10 and NF-kB for BPH.

\begin{tabular}{lcc}
\hline Parameters & AKR1B10 & NF-אB \\
\hline Cut-off & 0.955 & 0.840 \\
Sensitivity (\%) & 71.83 & 75.35 \\
Specificity (\%) & 97.86 & 98.57 \\
AUC & 0.857 & 0.831 \\
Std. Error & 0.025 & 0.029 \\
95\% CI & $0.809-0.906$ & $0.774-0.888$ \\
P-value & $<0.001$ & $<0.001$ \\
\hline
\end{tabular}

AKR1B10, aldo-keto reductase family 1 member B10; BPH, benign prostatic hyperplasia; AUC, area under the curve; Std., standard; CI, confidence interval.

the cut-off value was 0.955 , AKR1B10 had a sensitivity of $71.83 \%$ and a specificity of $97.86 \%$ for predicting $\mathrm{BPH}$; when the cut-off value was 0.840 , the sensitivity and specificity of NF- $\mathrm{kB}$ mRNA were 75.35 and $98.57 \%$ (Table III and Fig. 1).

Correlation between AKRIB10 in peripheral blood and clinical indicators. PSA, EGF, IL-6 and TNF- $\alpha$ in the peripheral blood of the research group were dramatically higher than those of the control group $(\mathrm{P}<0.001)$. Pearson correlation coefficient analysis demonstrated that AKR1B10 in the research group was positively correlated with PSA, EGF, IL-6 and TNF- $\alpha(r=0.704,0.415,0.745,0.742$, respectively; $\mathrm{P}<0.001$; Fig. 2).

Comparison of $A K R 1 B 10$ and $N F-\kappa B$ expression in rats. For the 10 modeling rats, all were successfully modeled, with a modeling rate of $100.0 \%$. There was no peritonitis observed in the rats. AKR1B10 and NF- $\mathrm{BB}$ mRNA levels in the prostate tissue of rats in the model group were obviously higher than those in the normal group $(\mathrm{P}<0.001)$. The positive rates of AKR1B10 and NF- $\mathrm{KB}$ in tissues were also markedly higher than those in the normal group $(\mathrm{P}<0.001)$. Semi-quantitative analysis of tissue sections revealed that the model group was higher than the control group ( $\mathrm{P}<0.001$; Figs. 3 and 4$)$.

Effect of AKRIBIO on the biological behavior of prostate hyperplasia cells. AKR1B10 was transfected into the BPH-1 cell line and the expression in AKR1B10-mimics, AKR1B10-inhibitor and AKR1B10-NC was detected. It was revealed that the AKR1B10-mimics group was dramatically higher than the other two groups (AKR1B10-NC and AKR1B10-inhibitor), while the AKR1B10-inhibitor group exhibited the lowest expression $(\mathrm{P}<0.001)$. According to the CCK-8 experiment, the cell proliferation of the AKR1B10-mimics group was the highest among the three groups $(\mathrm{P}<0.001)$. Flow cytometric analysis revealed that the apoptosis rate in the AKR1B10-mimics group was the lowest among the three groups, and the apoptosis rate in 

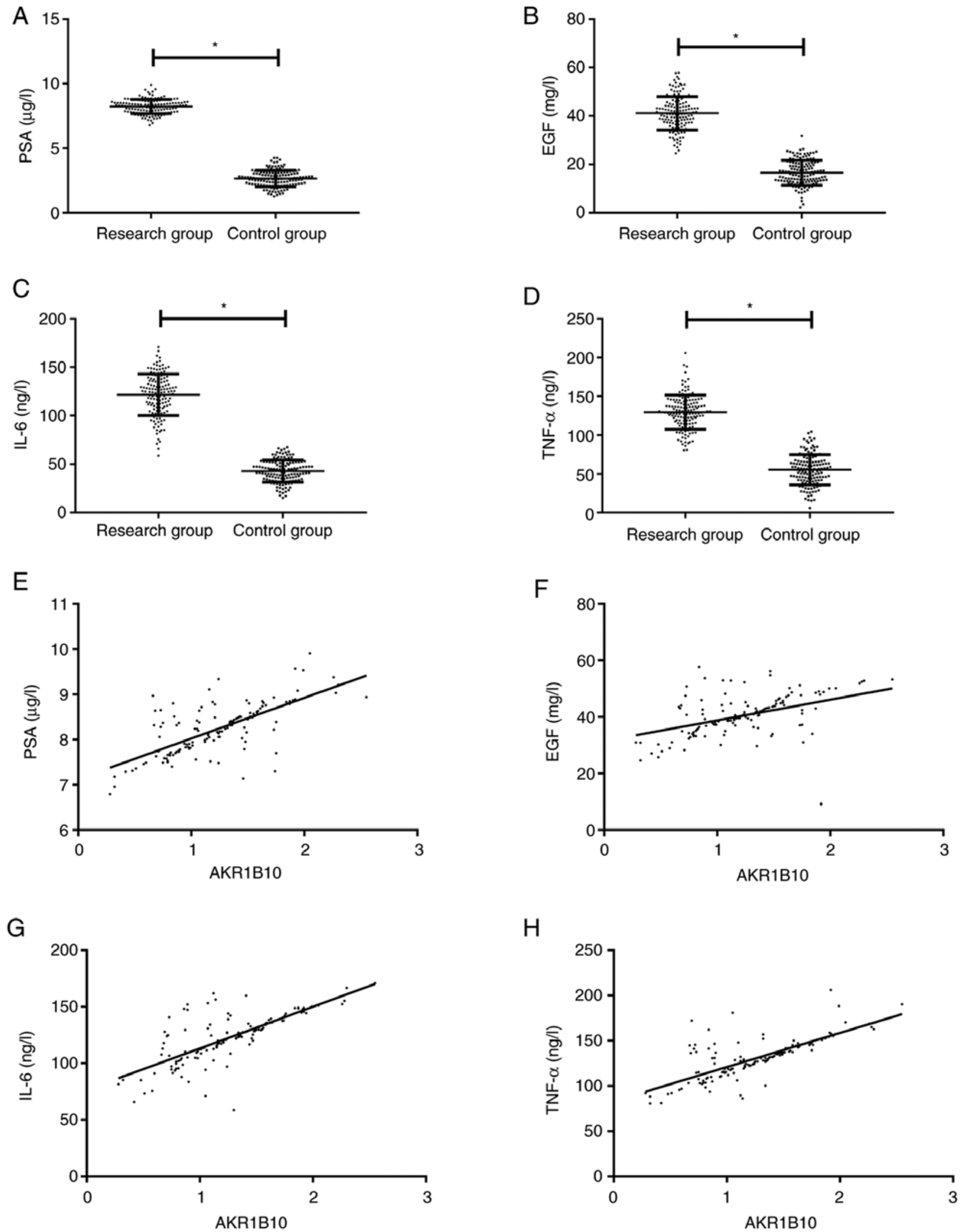

Figure 2. Correlation between AKR1B10 in peripheral blood and clinical indicators. (A) The PSA expression in the peripheral blood was compared. It was revealed that its level in the research group was significantly higher than that in the control group ( $\mathrm{P}<0.001)$. (B) The EGF expression in the peripheral blood was compared. It was revealed that its level in the research group was significantly higher than that in the control group ("P<0.001). (C) The IL-6 expression in the peripheral blood was compared. It was revealed that its level in the research group was significantly higher than that in the control group ("P<0.001). (D) The TNF- $\alpha$ expression in the peripheral blood was compared. It was revealed that its level in the research group was significantly higher than that in the control group $(" \mathrm{P}<0.001)$. (E) The correlation analysis between AKR1B10 and PSA in peripheral blood revealed a positive correlation $(\mathrm{r}=0.704)$. $(\mathrm{F}) \mathrm{The}$ correlation analysis between AKR1B10 and EGF in peripheral blood revealed a positive correlation $(\mathrm{r}=0.415)$. $(\mathrm{G})$ The correlation analysis of AKR1B10 and IL-6 in peripheral blood revealed a positive correlation $(\mathrm{r}=0.745)$. $(\mathrm{H})$ The correlation analysis of AKR1B10 and TNF- $\alpha$ in peripheral blood revealed a positive correlation ( $\mathrm{r}=0.742)$. AKR1B10, aldo-keto reductase family 1 member B10; PSA, prostate specific antigen; EGF, epidermal growth factor; IL, interleukin; $\mathrm{TNF}$, tumor necrosis factor. 
A
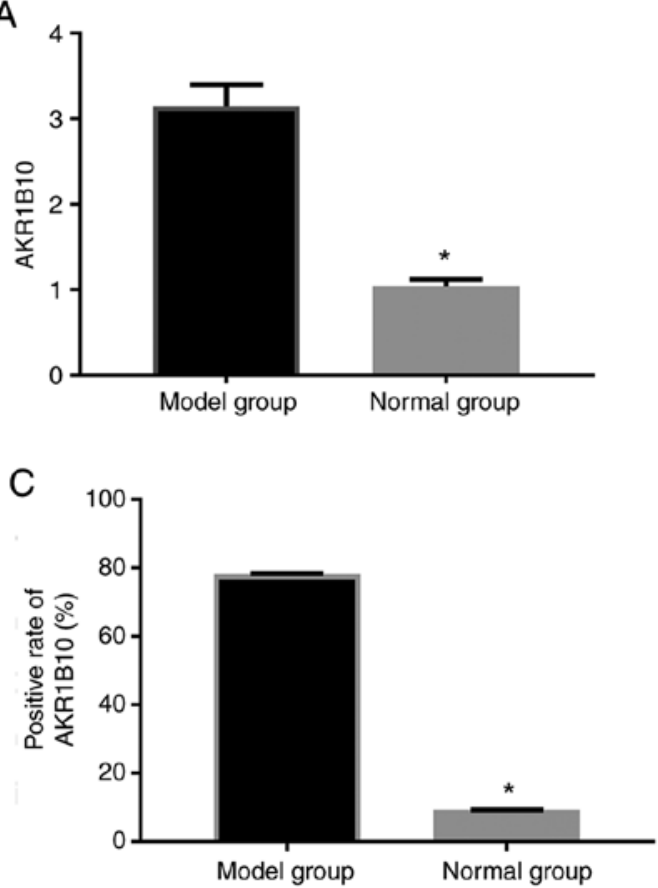

B
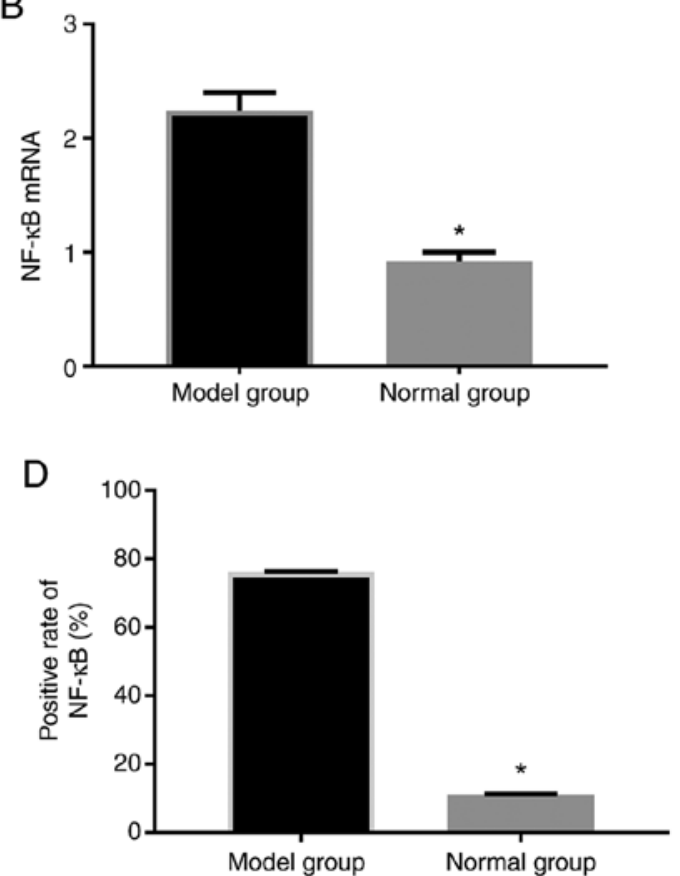

E

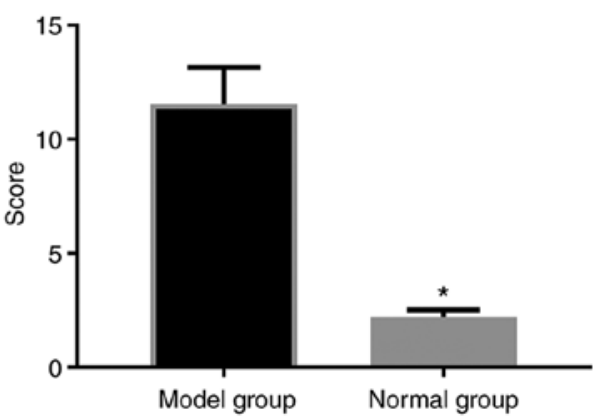

Figure 3. Comparison of AKR1B10 and NF-kB expression levels in rats. (A) Compared with the normal group, AKR1B10 in the prostate tissues of rats was significantly higher in the model group ( $\mathrm{P}<0.001)$. (B) The mRNA of NF- $\kappa \mathrm{B}$ in the prostate tissues of rats was compared. It was revealed that the NF- $\mathrm{kB}$ mRNA in the model group was significantly higher than that in the control group $\left({ }^{*} \mathrm{P}<0.001\right)$. (C) The positive staining rate of AKR1B10 in prostate tissues of rats was compared. It was revealed that the rate in the model group was significantly higher than that in the control group ("P $<0.001)$. (D) The positive staining rate of NF- $\mathrm{kB}$ in prostate tissues of rats was compared. It was revealed that the rate in the model group was significantly higher than that in the control group $\left({ }^{*} \mathrm{P}<0.001\right)$. (E) The semi-quantitative result staining scores of prostate tissue sections were compared. It was revealed that the score of the model group was significantly higher than that of the control group ("P<0.001). AKR1B10, aldo-keto reductase family 1 member B10.

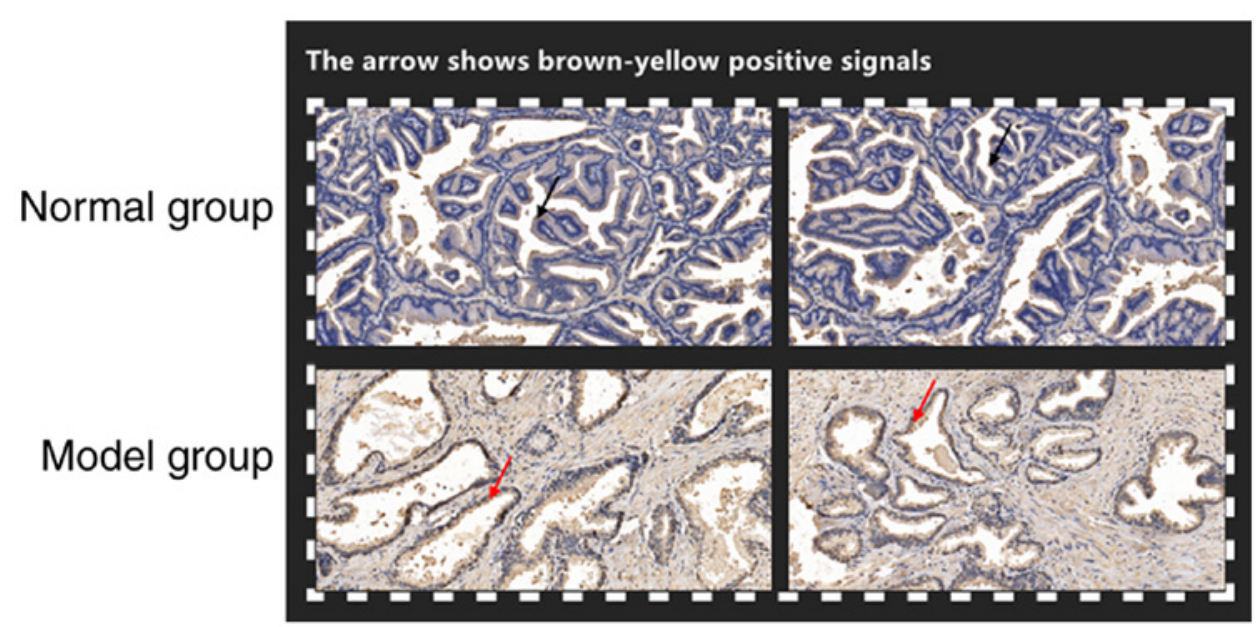

Figure 4. Results of tissue section examination (x200). The upper images reveal the tissue from rats in the normal group, and the lower images reveal the tissue of rats in the model group (two sections are presented for both the normal and model groups). AKR1B10 was mainly expressed in the cytoplasm of prostate epithelial cells in this sample. Among them, only a small number of brown-yellow positive signals (black arrows) were found in the epithelial cells of the normal group, and a large number of brown-yellow positive signals (red arrows) were found in the epithelial cells of the model group. AKR1B10, aldo-keto reductase family 1 member B10. 

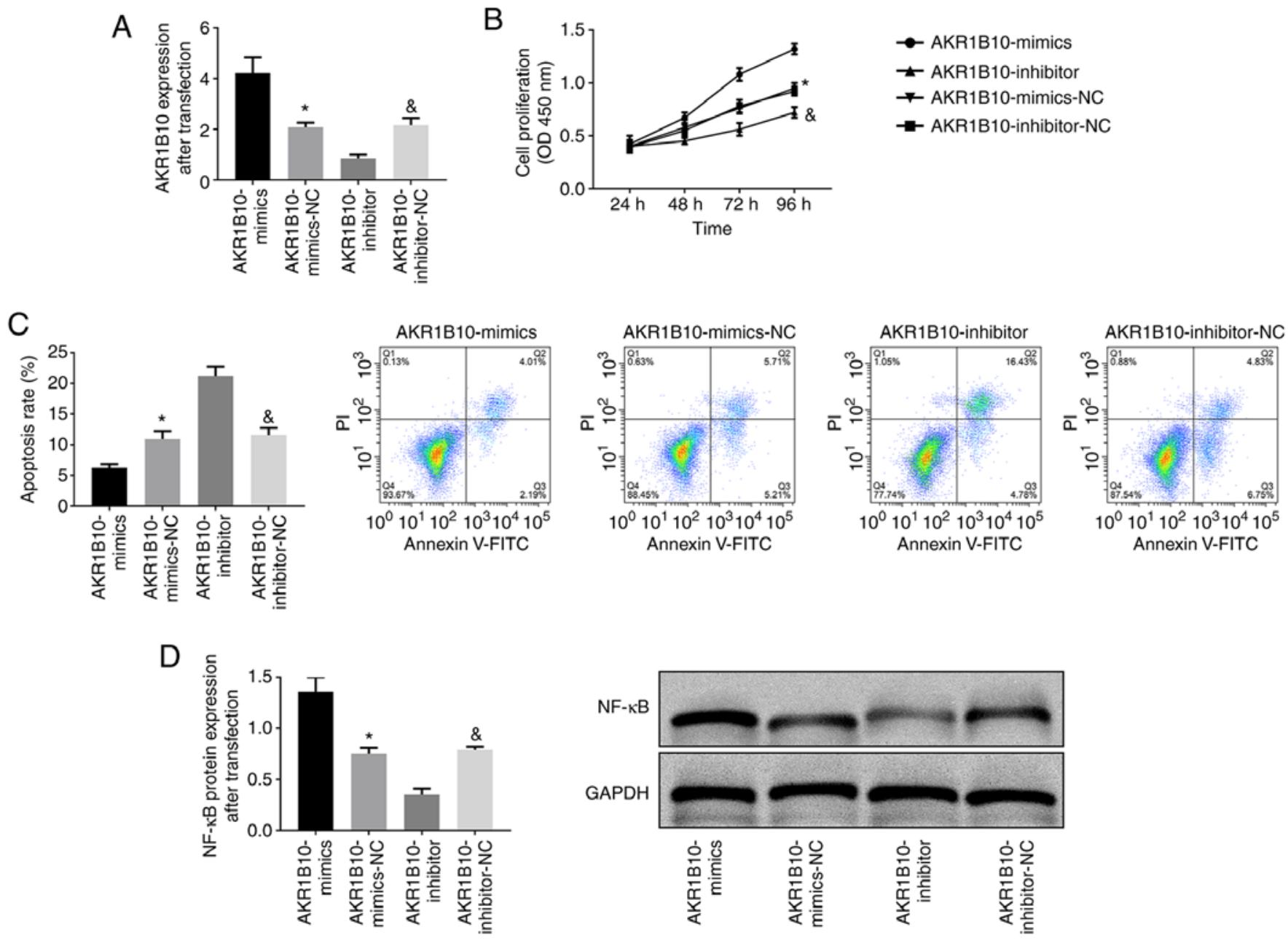

Figure 5. Effects of AKR1B10 on proliferation, apoptosis and NF-kB protein of BPH cells. (A) AKR1B10 expression after transfection of AKR1B10-mimics, AKR1B10-inhibitor and the corresponding AKR1B10-NC groups into the BPH-1 cell line. (B) Cell proliferation after transfection of AKR1B10-mimics, AKR1B10-inhibitor and the corresponding AKR1B10-NC groups into the BPH-1 cell line. (C) Apoptosis after transfection of AKR1B10-mimics, AKR1B10-inhibitor and the corresponding AKR1B10-NC groups into the BPH-1 cell line. (D) NF- $\kappa$ B protein expression after transfection of AKR1B10-mimics, AKR1B10-inhibitor and the corresponding AKR1B10-NC groups into the BPH-1 cell line. " $\mathrm{P}<0.05$, compared with AKR1B10-mimics group; ${ }^{*} \mathrm{P}<0.05$, compared with AKR1B10-inhibitor group.

the AKR1B10-inhibitor group was the highest $(\mathrm{P}<0.001)$. Western blotting revealed that the NF- $\kappa \mathrm{B}$ protein expression in the AKR1B10-mimics group increased, while that in the AKR1B10-inhibitor group decreased $(\mathrm{P}<0.001 ;$ Fig. 5).

\section{Discussion}

$\mathrm{BPH}$ is currently one of the most common male diseases among middle-aged and elderly men in the world. With the increasingly serious aging of the global population, the morbidity of $\mathrm{BPH}$ is also rising (16). Understanding BPH pathogenesis is quite marked for future prevention and treatment. AKR1B10, belonging to a class of carbonyl compounds that reduces aldehydes and ketones, not only has a protective effect on cells when they suffer from carbonyl toxicity damage, but can also stabilize acetyl-CoA carboxylase $\alpha$, block its degradation process through the ubiquitination pathway, and cause lipid synthesis in tumor cells (17). Previous studies revealed that AKR1B10 acted as a cancer-promoting factor in gastric cancer and breast cancer $(18,19)$. However, its role in BPH is ambiguous. The present study is aimed to explore the role of
AKR1B10 in BPH and reveal its mechanism influencing the biological behavior through NF-kB.

It demonstrated that the levels of AKR1B10 and NF- $\kappa \mathrm{B}$ mRNA in peripheral blood of BPH patients in the research group were dramatically higher than those in the control group, suggesting that the two may be involved in BPH development and progression. In fact, when Ko et al (20) and Hung et al (21) studied the effects of AKR1B10 on oral cancer and lung adenocarcinoma, they revealed that AKR1B10 was also markedly overexpressed, which supports our present results. However, Sinreih et al (22) demonstrated that AKR1B10 was markedly reduced in endometrial cancer and speculated that it had different effects in different diseases. AKR1B10 is a soluble monomer oxidoreductase in the cytoplasm, which can catalyze various endogenous and exogenous aldosterone-dependent NADH reduction reactions, mainly existing in embryonic liver and hepatocellular carcinoma tissues (23). However, ROC curve analysis revealed that AKR1B10 had a good predictive value for BPH occurrence and was positively associated with PSA, EGF, IL-6, TNF- $\alpha$ in the research group, which further confirmed the important influence of AKR1B10 on BPH 
occurrence. PSA, as the most sensitive indicator to reflect prostate state, is currently the preferred marker for diagnosing prostate cancer (24), and its physiological function is mainly to prevent semen coagulation. Usually the level in healthy individuals is extremely low, but PSA markedly increases once prostate disease or urogenital system disease occurs (25). However, AKR1B10 was also revealed to increase with PSA increase, which demonstrated that it had better monitoring value for BPH development. Moreover, AKR1B10 is better than PSA in that it has higher specificity and its increase is not as marked as PSA, which also suggests that it can be used as a reference indicator for future occurrence and development of BPH clinically.

To further verify the relationship between AKR1B10, $\mathrm{NF}-\kappa \mathrm{B}$ and $\mathrm{BPH}$, a BPH rat model was established and it was observed by section staining that the prostate tissue of rats exhibited a positive staining rate of AKR1B10 and NF- $\kappa \mathrm{B}$ compared to the tissue obtained from the normal group of rats. In addition, it was also determined that the prostate tissue of the BPH rat model had markedly higher mRNA expression of AKR1B10 and NF- $\kappa \mathrm{B}$ than that of normal rats. Furthermore, by transfecting AKR1B10 into prostate hyperplasia cells, we found that inhibiting AKR1B10 expression reduced the proliferation and apoptosis rates of prostate hyperplasia cells, and the protein expression of $\mathrm{NF}-\kappa \mathrm{B}$ also decreased. Therefore, it was inferred that the mechanism involved in the effect of AKR1B10 on BPH may be through NF- $\kappa$ B regula-

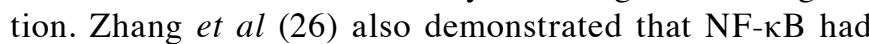
a promoting effect on prostate cancer, which supported our experimental results.

The present study was designed to explore the influence and mechanism of AKR1B10 on BPH. However, due to the limited experimental conditions, deficiencies remain. The present study focused on the influence of AKR1B10 on BPH through $\mathrm{NF}-\kappa \mathrm{B}$, but it does not exclude the possibility of other influences through other pathways, which will be a key direction of our future research. Moreover, due to the short experimental period, it is impossible to ascertain how AKR1B10 affects the long-term prognosis of BPH. We will conduct a more in-depth and comprehensive analysis of the aforementioned limitations to obtain more concrete experimental results.

In summary, high expression of AKR1B10 in BPH promoted the proliferation and reduced the apoptosis of prostate cells, and its mechanism might be through the regulation of NF- $\mathrm{NB}$.

\section{Acknowledgements}

Not applicable.

\section{Funding}

The present study was supported by the National Natural Science Foundation of China (grant no. 81603634), the Hunan Male Diseases Clinical Medical Research Center of Traditional Chinese Medicine (grant no. 2018SK4012), the Hunan Clinical Medical Technology Innovation Guidance Plan (grant no. 2017SK50304) and the Hunan Scientific Research Project of Traditional Chinese Medicine (grant no. 201732).

\section{Availability of data and materials}

The datasets used and/or analyzed during the present study are available from the corresponding author on reasonable request.

\section{Authors' contributions}

WX designed and conceived the study and wrote the manuscript. YG performed the PCR, ELISA and western blotting experiments. JZ was responsible for CCK-8 detection and flow cytometry. RZ analyzed and interpreted the data of patients. QC helped with the statistical analysis. All authors read and approved the final manuscript.

\section{Ethics approval and consent to participate}

The present study was approved by the Ethics Committee of the First Hospital of Hunan University of Chinese Medicine (Changsha, China). Patients who participated in this research, signed the informed consent and had complete clinical data. Signed written informed consents were obtained from the patients.

\section{Patient consent for publication}

Not applicable.

\section{Competing interests}

The authors declare that they have no competing interests.

\section{References}

1. Chughtai B, Forde JC, Thomas DD, Laor L, Hossack T, Woo HH, Te AE and Kaplan SA: Benign prostatic hyperplasia. Nat Rev Dis Primers 2: 16031, 2016.

2. Egan KB: The epidemiology of benign prostatic hyperplasia associated with lower urinary tract symptoms: Prevalence and incident rates. Urol Clin North Am 43: 289-297, 2016.

3. Lee YJ, Lee JW, Park J, Seo SI, Chung JI, Yoo TK and Son H: Nationwide incidence and treatment pattern of benign prostatic hyperplasia in Korea. Investig Clin Urol 57: 424-430, 2016.

4. Kim EH, Larson JA and Andriole GL: Management of benign prostatic hyperplasia. Annu Rev Med 67: 137-151, 2016.

5. Lim KB: Epidemiology of clinical benign prostatic hyperplasia. Asian J Urol 4: 148-151, 2017.

6. Al-Khalil S, Boothe D, Durdin T, Sunkara S, Watkins P Yang S, Haynes A and de Riese W: Interactions between benign prostatic hyperplasia $(\mathrm{BPH})$ and prostate cancer in large prostates: A retrospective data review. Int Urol Nephrol 48: 91-97, 2016.

7. Khazaei S, Rezaeian S, Ayubi E, Gholamaliee B, Pishkuhi MA, Khazaei S, Mansori K, Nematollahi S, Sani M and Hanis SM: Global prostate cancer incidence and mortality rates according to the human development index. Asian Pac J Cancer Prev 17: 3793-3796, 2016.

8. Corona G, Tirabassi G, Santi D, Maseroli E, Gacci M, Dicuio M, Sforza A, Mannucci E and Maggi M: Sexual dysfunction in subjects treated with inhibitors of $5 \alpha$-reductase for benign prostatic hyperplasia: A comprehensive review and meta-analysis. Andrology 5: 671-678, 2017.

9. Zhang L, Wu B, Zha Z, Zhao H, Yuan J, Jiang Y and Yang W: Surgical margin status and its impact on prostate cancer prognosis after radical prostatectomy: A meta-analysis. World J Urol 36: 1803-1815, 2018.

10. Macey MR and Raynor MC: Diagnosis of benign prostatic hyperplasia. Chapter 2. In: Prostatic Artery Embolization. Isaacson AJ, Bagla S, Raynor MC and $\mathrm{Yu} \mathrm{H}$ (eds). Springer, Cham, pp11-19, 2020. 
11. He YC, Shen Y, Cao Y, Tang FQ, Tian DF, Huang CF, Tao H Zhou FL, Zhang B, Song L, et al: Overexpression of AKR1B10 in nasopharyngeal carcinoma as a potential biomarker. Cancer Biomark 16: 127-135, 2016.

12. Wang YY, Qi LN, Zhong JH, Qin HG, Ye JZ, Lu SD, Ma L, Xiang BD, Li LQ and You XM: High expression of AKR1B10 predicts low risk of early tumor recurrence in patients with hepatitis B virus-related hepatocellular carcinoma. Sci Rep 7: 42199, 2017.

13. Reddy KA, Kumar PU, Srinivasulu M, Triveni B, Sharada K, Ismail A and Reddy GB: Overexpression and enhanced specific activity of aldoketo reductases (AKR1B1 \& AKR1B10) in human breast cancers. Breast 31: 137-143, 2017.

14. Nishinaka T, Miura T, Shimizu K and Terada T: Identification and characterization of functional antioxidant response elements in the promoter of the aldo-keto reductase AKR1B10 gene. Chem Biol Interact 276: 160-166, 2017.

15. Khan S, Bennit HF and Wall NR: The emerging role of exosomes in survivin secretion. Histol Histopathol 30: 43-50, 2015.

16. Livak KJ and Schmittgen TD: Analysis of relative gene expression data using real-time quantitative PCR and the 2(-Delta Delta C(T)) method. Methods 25: 402-408, 2001.

17. Ishola IO, Yemitan $\mathrm{KO}$, Afolayan OO, Anunobi $\mathrm{CC}$ and Durojaiye TE: Potential of Moringa oleifera in the treatment of benign prostate hyperplasia: Role of antioxidant defence systems. Med Princ Pract 27: 15-22, 2018

18. Cao Z, Zhou B, Chen X, Huang D, Zhang X, Wang Z, Huang H, Wang Y and Cao D: Statil suppresses cancer cell growth and proliferation by the inhibition of tumor marker AKR1B10. Anticancer Drugs 25: 930-937, 2014.

19. Yao HB, Xu Y, Chen LG, Guan TP, Ma YY, He XJ, Xia YJ, Tao HQ and Shao QS: AKR1B10, a good prognostic indicator in gastric cancer. Eur J Surg Oncol 40: 318-324, 2014.
20. Ko HH, Cheng SL, Lee JJ, Chen HM, Kuo MY and Cheng SJ: Expression of AKR1B10 as an independent marker for poor prognosis in human oral squamous cell carcinoma. Head Neck 39: 1327-1332, 2017

21. Hung JJ, Yeh YC and Hsu WH: Prognostic significance of AKR1B10 in patients with resected lung adenocarcinoma. Thorac Cancer 9: 1492-1499, 2018.

22. Sinreih M, Štupar S, Čemažar L, Verdenik I, Frković Grazio S, Smrkolj S and Rižner TL: STAR and AKR1B10 are down-regulated in high-grade endometrial cancer. J Steroid Biochem Mol Biol 171: 43-53, 2017.

23. Taskoparan B, Seza EG, Demirkol S, Tuncer S, Stefek M, Gure AO and Banerjee S: Opposing roles of the aldo-keto reductases AKR1B1 and AKR1B10 in colorectal cancer. Cell Oncol (Dordr) 40: 563-578, 2017.

24. Preston MA, Batista JL, Wilson KM, Carlsson SV, Gerke T, Sjoberg DD, Dahl DM, Sesso HD, Feldman AS, Gann PH, et al: Baseline prostate-specific antigen levels in midlife predict lethal prostate cancer. J Clin Oncol 34: 2705-2711, 2016.

25. Filella $X$ and Foj L: Prostate cancer detection and prognosis: From prostate specific antigen (PSA) to exosomal biomarkers. Int J Mol Sci 17: 1784, 2016.

26. Zhang L, Zhang Q, Li L, Wang Z, Ying J, Fan Y, He Q, Lv T, Han W, Li J, et al: DLEC1, a 3p tumor suppressor, represses $\mathrm{NF}-\kappa \mathrm{B}$ signaling and is methylated in prostate cancer. J Mol Med (Berl) 93: 691-701, 2015.

(i) $\mathrm{E}$ This work is licensed under a Creative Common Attribution-NonCommercial-NoDerivatives 4.0 International (CC BY-NC-ND 4.0) License. 